\title{
The Smoothing Filter for Mobile Augmented Reality Using the Moving Average
}

\author{
Piyapong Dangkham ${ }^{+}$ \\ Information and Communication Engineering, Faculty of Industrial Technology, Thepsatri Rajabhat \\ University, Thailand
}

\begin{abstract}
This research presents the use of moving average to calculate the smooth data for mobile augmented reality on web application with HTML5. The advantage of HTML5 is that the users can access the application immediately without installation application required. The process of implementation mobile augmented reality are access the mobile camera, getting GPS data and handling orientation data. The user's viewpoint is calculated in the handling orientation data from the sensor. It is based on the web browser and the sensor, the data in general is not free from noise and jitter. The moving average is used to smooth the time series data in the short-term fluctuations. The research use simple moving average and exponential moving average to estimate the smooth data. In the maximum different adjacent data at 5 , the root mean square error of simple moving average and exponential moving average are 4.63 and 4.14, respectively. The result shows that exponential moving average is better than simple moving average.
\end{abstract}

Keywords: augmented reality, HTML5, moving average.

\section{Introduction}

The Augmented Reality (AR) is a technology which allows the user to see the real world with the virtual objects. The commercial mobile AR applications already exist (e.g. Layar, Wikitude and Aurasma) but they all require installation on the user's device. It is not suitable for the user. They have to install the specific application for each usage. The HTML5 is a technology which can be allowed in a cross-platform manner. The HTML5 supports the developer to use the mobile device sensors. The paper in [1] develops the marker based mobile AR application with HTML5, webRTC and XML3D. The application is a cross-platform supported web browser. In [2], the framework for mobile web application using HTML5 is presented. The framework can be used for the implementation of mobile web application with the marker based. The benefit of HTML5 is that users can use the application without installation required.

The processes to develop the augmented reality with the location based on web application are access the device camera, getting GPS data and handling orientation data. The tilt of the device is captured to be used for a direction of the user's viewpoint. The HTML5 events allow the access of the sensor information. The implementation of these events varies. It is based on the web browser and the sensor, the data in general is not free from noise and jitter. The filter steps need to be applied. There are many types of filtering [3] such as the different interpolating polynomials method, splines, Fourier transform and wavelet transform. Some filter requires high computational and increases the processing time but the system needs to be in real-time. The filter, which is called smoothing filter, results in smoother data over time. The exponential moving average (EMA) is a moving average filter which has also used by different researchers for smoothing filter [4-6]. The EMA is good combination of low computational cost and relatively high accuracy.

\footnotetext{
Corresponding author. Tel.: +66890031265; fax: +6636422125.

E-mail address: piyapong.d@ lawasri.tru.ac.th.
} 
This paper presents the smoothing orientation data from the mobile sensor using the moving average filter. This process enhances the user's viewpoint movement which is one of steps for development the location based mobile augmented reality with web application.

\section{Mobile AR with Location Based}

There are 3 processes [7] to develop the mobile AR with location based on web application: access the mobile camera, getting GPS data and handling orientation data.

- Access the Mobile Camera: The getUserMedia is a method of Navigation object which can support the developer for permission to use the camera. The live video is delivered to the <video > element in the specified success JavaScript callback function.

- Getting GPS Data: The Geolocation is a HTML5 API for access to the device geographical locations. The Coordinate object properties to get the geographic coordinate are latitude and longitude. They are measured in the decimal degrees. The GPS data is used to calculate a user's bearing. For the bearing, it is the angle between the North and the user's position to the POI (point of interest).

- Handling Orientation Data: The tilt of the device can be captured from the sensor with the HTML5 API, DeviceOrientation. There are 3 properties [8]: alpha, beta and gamma. The alpha property is a direction of the device according to the compass. The alpha angle can range between 0 and 360 degrees. The alpha is 0 when the top of the device is pointed directly to Earth's North Pole. The alpha property is used to be a direction of the user's viewpoint. The beta and gamma properties are the angle in degrees of the device which is tilted front-to-back and left-to-right, respectively. The range of beta is between -180 and 180 degrees. The range of gamma is between - 90 and 90 degrees. Fig. 1 shows the user's bearing to POI and the user's viewpoint which are calculated from GPS data and alpha property, respectively.

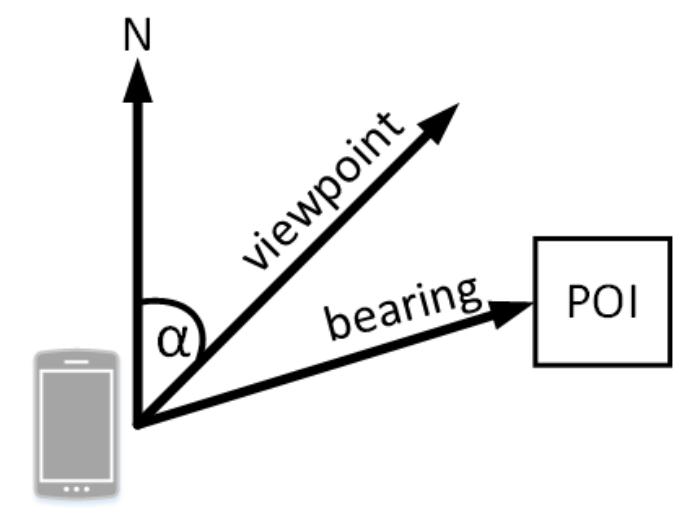

Fig. 1: The user's viewpoint and the user's bearing to POI.

\section{The Moving Average}

The moving average is used to smooth the time series data in the short-term fluctuations. A simple moving average (SMA) is a simple low-pass filter. It estimates the data by using sliding window. The equation for the n-sample (window size) SMA is [5]:

$$
S_{t}=\left(X_{t}+X_{t-1}+X_{t-2}+\ldots+X_{t-n+1}\right) / n
$$

where $S_{t}$ is the output of SMA and $X_{t}$ is the raw data.

The Exponential Moving Average (EMA) is one of moving average commonly applied to smooth data. The EMA uses the exponential window function which assigns the exponentially decreasing weights over time. The general proportional weights are expanded to obtain: $\left[\begin{array}{lllll}1 & (1-\alpha) & (1-\alpha)^{2} & (1-\alpha)^{3} & \ldots\end{array}\right]$. The EMA output is given by equation below [3]:

$$
S_{t}=\alpha X_{t}+(1-\alpha) S_{t-1}
$$

where $S_{t}$ is the output of EMA, $X_{t}$ is the raw data, and $\alpha$ is the smoothing factor $(0<\alpha<1)$. The errors are specified as root mean square error (RMSE). The parameters can be estimated by minimizing the RMSE. 


\section{Experiment and Result}

The mobile AR application analyzes the tilt of mobile data for the criteria of user's bearing and the user's viewpoint. When the user changes viewpoint, the DeviceOrientation event will send the data from the sensor. The experiment is test on the SAMSUNG A7 mobile with Android 6. The application runs on SAMSUNG internet 5.4 (web browser) for handling the orientation data.

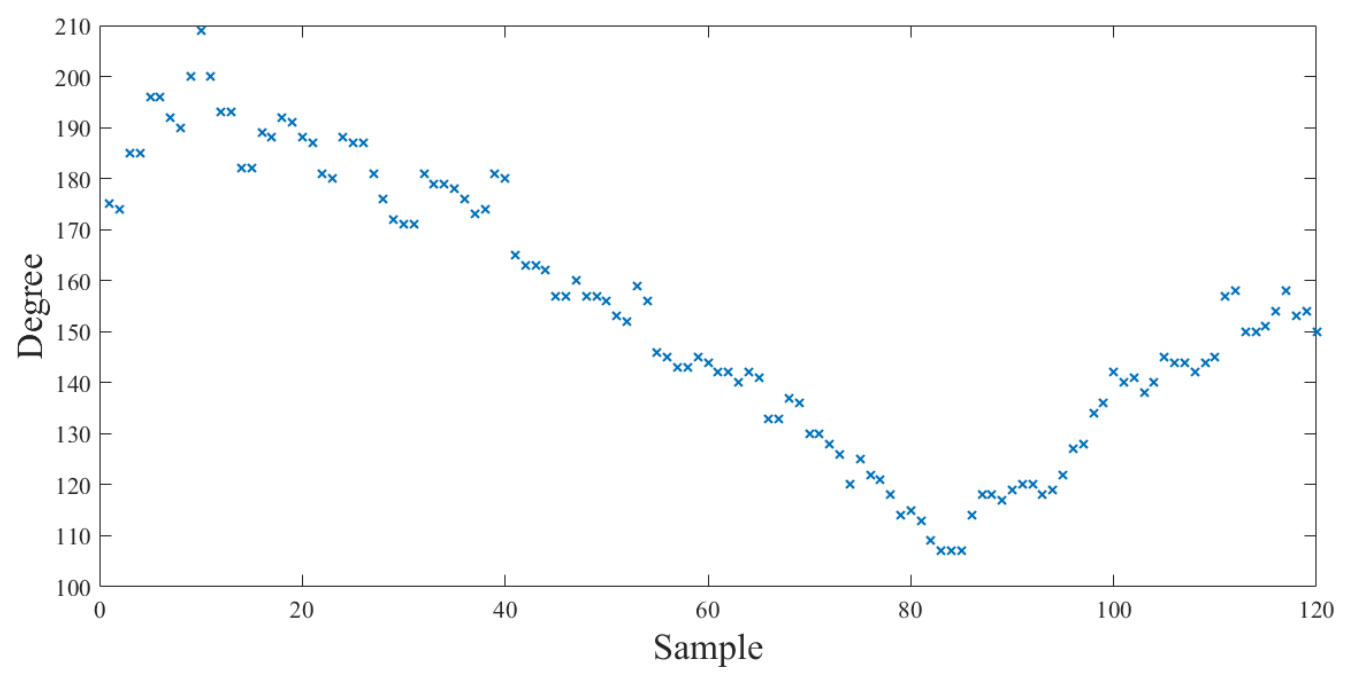

Fig. 2: The user's viewpoint.

Fig. 2 shows the data set of user's viewpoint from alpha property in DeviceOrientation event when the user focus on the South to the East $(90<\alpha<180)$. There are 120 samples. The maximum different adjacent data $\left(\Delta S_{t}=\left|S_{t}-S_{t-1}\right|\right)$ is 15 degree. It shows that user's viewpoint changes rapidly. The mobile AR data will blink, display and disappear alternately. The data need to be smooth. In Fig. 3 shows the results of using SMA with window size are 3, 5, 7 and 9 to calculate smooth data.

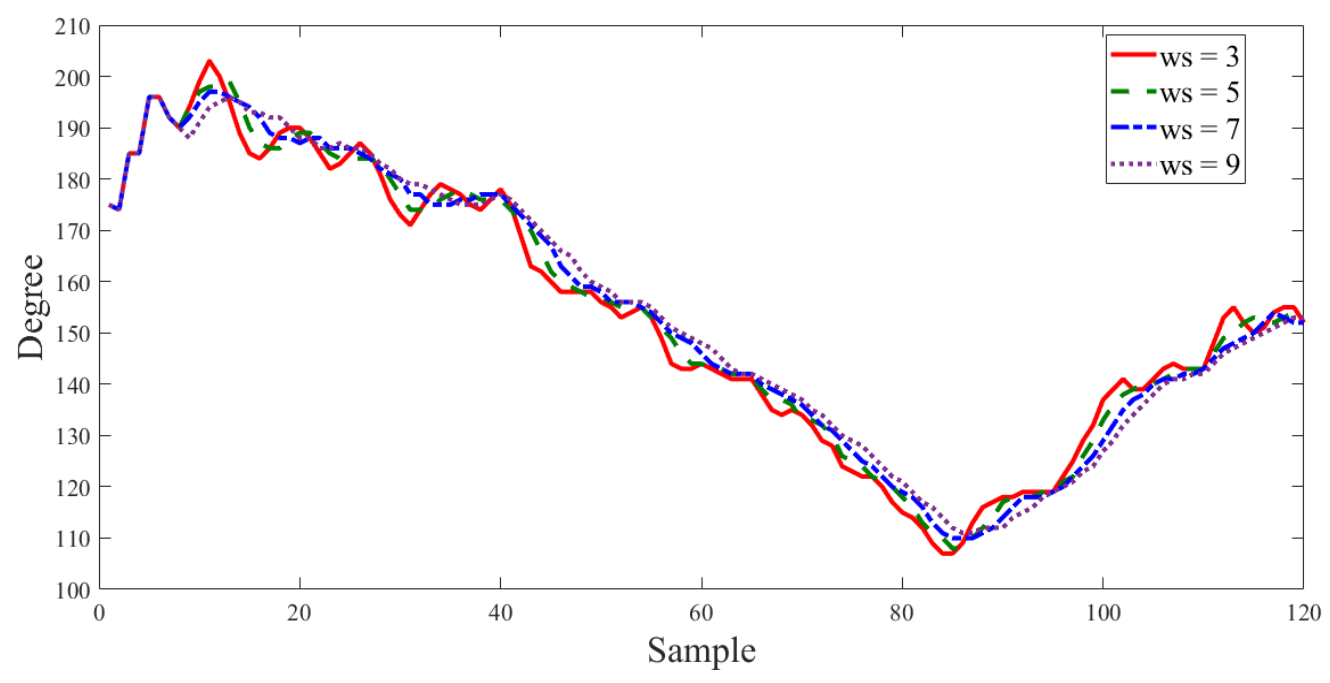

Fig. 3: The SMA result.

Table 1: The SMA performance.

\begin{tabular}{|c|c|c|}
\hline window size & $\max \left(\Delta S_{t}\right)$ & RMSE \\
\hline 3 & 6 & 3.30 \\
\hline 5 & 5 & 4.63 \\
\hline 7 & 4 & 5.81 \\
\hline 9 & 3 & 6.44 \\
\hline
\end{tabular}


In Table 1 , the results show that the largest $\max \left(\Delta S_{t}\right)$ is 6 at the window size is 3 and the smallest $\max \left(\Delta S_{t}\right)$ is 3 at the window size is 9 . The window size at 9 is more smooth data than window size at 3 because the bigger window size calculates the average from more samples. But the smooth data has worse RMSE because the raw data is more fluctuation. The EMA is used to calculate the smooth data. The results show in Fig. 4 and Table 2. The $\alpha$ is varied at $0.1,0.2,0.3$, and 0.4 . When the $\alpha$ is smaller, the data estimated is more smooth. It is the effect of the proportional weight. If the $\alpha$ is smaller, the previous EMA $\left(S_{t-1}\right)$ is more weight. The same as SMA, the smooth data has worse RMSE.

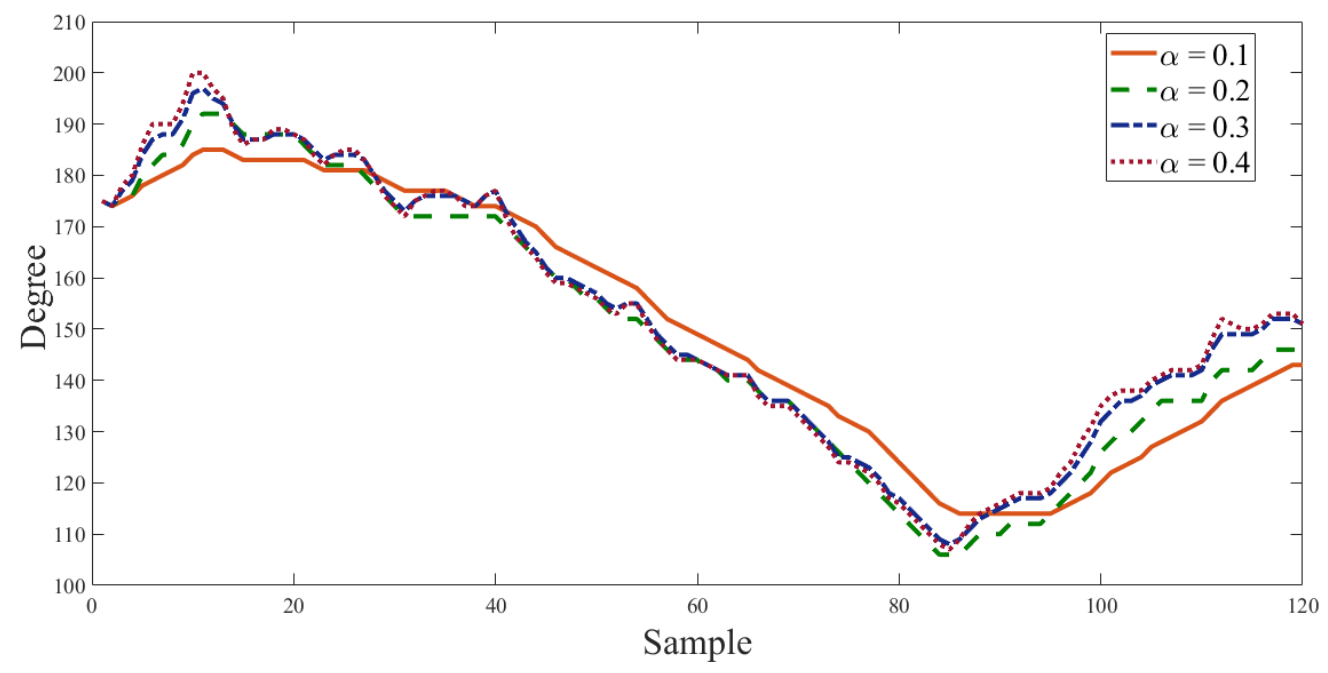

Fig. 4: The EMA result.

Table 2: The EMA performance.

\begin{tabular}{|c|c|c|}
\hline smoothing factor $(\alpha)$ & $\max \left(\Delta S_{t}\right)$ & RMSE \\
\hline 1 & 2 & 9.86 \\
\hline 2 & 4 & 5.63 \\
\hline 3 & 5 & 4.14 \\
\hline 4 & 6 & 3.17 \\
\hline
\end{tabular}

In Table 3, the comparative RMSE of SMA and EMA are shown. At the same $\max \left(\Delta S_{t}\right)$, the RMSE of EMA is smaller than SMA. It shows that EMA is suitable for estimating smooth data. The advantage of EMA is using 2 data: raw data $\left(X_{t}\right)$ and previous EMA $\left(S_{t-1}\right)$, while SMA used $n-1$ previous data.

Table 3: The comparative RMSE of SMA and EMA.

\begin{tabular}{|c|c|c|}
\hline \multirow{2}{*}{$\max \left(\Delta S_{t}\right)$} & \multicolumn{2}{|c|}{ RMSE } \\
\cline { 2 - 3 } & SMA & EMA \\
\hline 4 & 5.81 & 5.63 \\
\hline 5 & 4.63 & 4.14 \\
\hline 6 & 3.30 & 3.17 \\
\hline
\end{tabular}

\section{Conclusion}

In this research, the moving average is used to smooth the orientation data of mobile device. The orientation data are used to be the user's viewpoint. If the user's viewpoint changes rapidly, the mobile AR data will blink, display and disappear alternately. This research enhances the user's viewpoint movement which is one of steps for development the location based mobile augmented reality with web application. The simple moving average (SMA) and exponential moving average (EMA) are used to estimate the smooth data. The SMA estimates the data by using sliding window with the n-sample. The EMA uses the exponentially decreasing weights over time. The smooth data can be estimated by minimizing the root mean 
square error (RMSE) and the maximum different adjacent data. At the same maximum different adjacent data, the RMSE of SMA is more than the EMA. The result shows that EMA is better than SMA.

\section{References}

[1] A. Karhu, A. Heikkinen and T. Koskela, "Towards augmented reality applications in a mobile web context," 8th International Conference on Next Generation Mobile Apps, 2014, pp. 1-6.

[2] C. Bouras, A. Papazois and N. Stasinos, "A framework for cross-platform mobile web applications using HTML5," International Conference on Future Internet of Things and Cloud, 2014, pp. 420-424.

[3] B. Alexander, T. Ivan and B. Denis, "Analysis of noisy signal restoration quality with exponential moving average filter," International Siberian Conference on Control and Communications (SIBCON), Moscow, 2016, pp. 1-4.

[4] M. Adjeisah, Yi Yang and Lian Li, "Joint Filtering: Enhancing gesture and mouse movement in Microsoft Kinect application," 12th International Conference on Fuzzy Systems and Knowledge Discovery (FSKD), Zhangjiajie, 2015, pp. 2528-2532.

[5] S. M. Shaikh, M. S. A. Hossain, T. B. Zaman, U. B. Tayab and M. A. Humayun, "Application of energy capacitor system with minimum energy storage capacity for smoother frequency and output power of a grid connected wind farm," 3rd International Conference on Electrical Information and Communication Technology (EICT), Khulna, 2017, pp. 1-6.

[6] A. Hammoudeh and L. Al-Sharif, "Estimating the elevator traffic system arrival rate using exponentially weighted moving average(EWMA)," IEEE Jordan Conference on Applied Electrical Engineering and Computing Technologies (AEECT), Aqaba, 2017, pp. 1-5.

[7] Piyapong Dangkham, "Mobile augmented reality on web-based for the tourism using HTML5," International Conference on Information Networking (ICOIN), 2018, pp. 482-485.

[8] Jonas Etzold, Michael Englert, Paul Grimm, Yvonne Jung, and Marcel Klomann, "MIPos: towards mobile image positioning in mixed reality web applications based on mobile sensors," 19th International ACM Conference on $3 D$ Web Technologies, August 2014, pp. 17-25. 